\title{
Composite Auricular Chondrocutaneous Graft Is Useful For Lower Eyelid Reconstruction after Surgery for Cancer of the Maxilla
}

\author{
Hiroko Ochiai $^{1 *}$, Naoko Yagi ${ }^{1}$, Aiko Oka ${ }^{1}$, Eri Hirata ${ }^{1}$, Hanayo Yabuki ${ }^{1}$ and Masato Fujii ${ }^{2}$ \\ ${ }^{1}$ Department of Plastic and Reconstructive Surgery, National Hospital Organization Tokyo Medical Center, 2-5-1 Higashigaoka, Meguro-ku, Tokyo 152-8902, \\ Japan \\ ${ }^{2}$ Department of Otorhinolaryngology, National Tokyo Medical Center, 2-5-1 Higashigaoka, Meguro-ku, Tokyo 152-8902, Japan
}

\begin{abstract}
Ectropion of lower eyelid can occur after surgical resection of maxillary cancers. The present report describes three cases in which we used a composite auricular chondrocutaneous graft to address lower eyelid ectropion after subtotal maxillectomy. The tarsal strip procedure and lateral canthopexy is sometimes used for ectropion with facial nerve paresis to tighten the lower lid,but it is not always effective after maxillectomy. The lower eyelid is characterized by a thin musculocutaneous anterior lamella and a posterior lamella composed of the tarsus and conjunctiva. We harvested auricular cartilage along with 5-10 mm of cutaneous tissue. The composite graft was attached to the lower eyelid to provide posterior support and anterior skin. In all cases, the patient's complaints and objective symptoms were alleviated postoperatively. As a result, we think that ectropion is caused by 1) contracture and weight of grafted flaps, 2) contracture by Weber-Ferguson incision and 3) dysfunction of orbicularis oculi muscle. In addition, weak and thin tarsus was confirmed on surgery finding, it is predicted that the local vascularity is compromised after surgical resection of maxillary cancers. In conclusion, patients with this defect require reconstruction of both the anterior and posterior lamella. The present report suggests that an auricular chondrocutaneous graft is a suitable material for lower eyelid reconstruction after subtotal maxillectomy.
\end{abstract}

\section{Introduction}

Maxillectomy is the treatment of choice for management of malignant tumors of the maxilla. Reconstruction of such defects continues to be an extremely demanding challenge for plastic surgeons who aim to restore function and cosmetic appearance. Postoperatively, some patients can develop severe ectropion. This results in reduced protection for the eye and subsequent tearing, irritation, burning, itching, discomfort, and even decreased vision Moreover, the redness of the everted conjunctiva and sclera are conspicuous in appearance and undesirable. There is tendency that the symptoms become more seriousdepending on resection range, but a similar symptom really occurs after either subtotal maxillectomy(Figure 1) [1].

Lower eyelid reconstruction has been attempted using various materials, including fascia, preserved sclera, costal cartilage, nasal cartilage and ear cartilage [2-5]. Auto-grafting of the ear conchal cartilage may also be useful for this process, as the shape, curve and width of this graft closely resemble that of the tarsus of the eyelid. Use of skin grafting in tandem with cartilage grafting may also serve to lengthen the anterior lamella of the lid. The present report describes cases in which successful correction of moderate to severe lower lid ectropion (occurring after subtotal maxillectomy) was performed using an auricular chondrocutaneous graft.

\section{Case Reports}

\section{Case 1: 77-year-old male}

After subtotal resection of a left maxilla cancer by Weber-Ferguson incision, reconstruction of the surgical defect was performed using a free latissimusdorsi muscle flap. Two months after the operation, he was noted to have ectropion and lagophthalmos (Figure 2). Therefore, we performed a tarsal strip procedure with a fascia lata sling and nasolacrimal duct reconstruction. However, the patient did not experience any improvement in the ectropion.

Eight months later, further surgical management was elected. A skin incision was made on the back side of the auricle longitudinally (Figure 3a). The distance from the infraorbital rim to the cilia of the unaffected

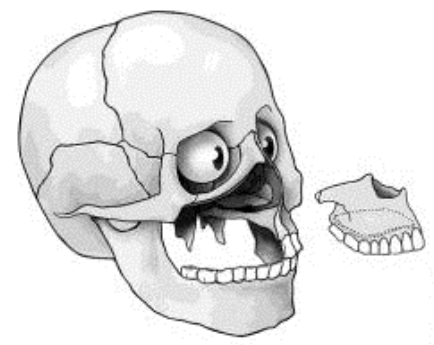

Figure 1. Type2 (subtotal maxillectomy) defect.

Correspondence to: Hiroko Ochiai, School of Medicine, Department of Plastic and Reconstructive Surgery, National Hospital Organization Tokyo Medical Center2-5-1 Higashigaoka, Meguro-ku, Tokyo 152-8902, Japan, Tel: +81-33411-0111; Fax: +81-3-3412-9811; E-mail: hirokoochiai@ntmc.hosp.go.jp

Key words: subtotal maxillectomy;ectropion; lower eyelid reconstruction; auricular chondrocutaneous graft

Received: November 15, 2016; Accepted: November 28, 2016; Published: December 30, 2016 


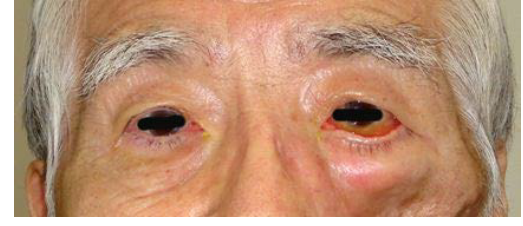

Figure 2. Preoperative case 1.

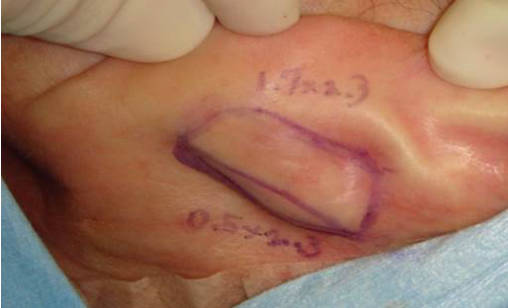

Figures 3a. Design of harvesting the composite auricular graft.

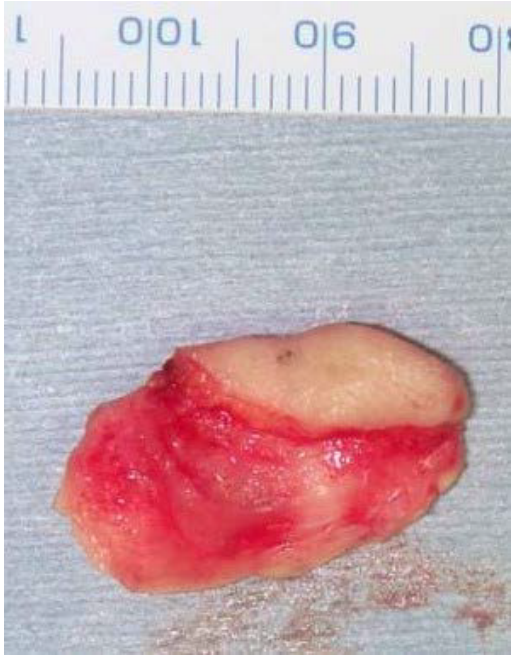

Figures 3b. The auricular chondrocutaneous graft.

site was measured. The same height of auricular cartilage $(15 \times 10 \mathrm{~mm})$ combined with a $3 \times 20 \mathrm{~mm}$ cutaneous region was harvested(Figure $3 \mathrm{~b}$ ). The donor site was sutured in line. Harvesting ear conchal cartilage from the retro-auricular area may avoid visible scarring. A subcilial incision was made, and the pretarsal layer was undermined to form a pocket for grafting tissue. The chondrocutaneous graft was placed under the orbicularis oculi muscle and was fixed to the infraorbital rim inferiorly and inserted into the pocket subjacent to the root of the cilia superiorly (Figure 4). The cutaneous portion of the graft was sutured to the edge of subcilial incision, forcing the lid backwards (Figure 5). Additional blepharoptosis operation was done at the same time.

After the correction, ectropion improved, and the post auricular scar was not visible (Figure 6\&7). Furthermore, the symptoms of epiphora and conjunctival discharge were alleviated, probably due to the nasolacrimal duct reconstruction.

\section{Case 2: 75-year-old female}

After subtotal resection of a left maxilla cancer by Weber-Ferguson incision, reconstruction of the surgical defect was performed using a free latissimusdorsi muscle flap. Even months postoperatively, she was noted to have ectropion and lagophthalmos (Figure 8). A tarsal strip procedure with fascia lata sling was performed, and the fascia lata was sutured to the ligament medially and to the bone membrane of the orbital rim laterally. Ectropion improved somewhat, but the cilial edge of the lower eyelid bent sharply outside, and the red color was not aesthetically pleasing (Figure 9). Therefore, auricular chondrocutaneous grafting was performed at 20 months after the first correction. A $17 \times 23$ size auricular cartilage graft combined with $5 \times 23$ $\mathrm{mm}$ of cutaneous tissue was harvested and grafted to the lower eyelid in a manner similar to that described for Case 1. Ectropion and eyelid bending subsequently improved (Figure 10).

\section{Case 3: 89-year-old female}

After subtotal resection of a right hard palate cancer by WeberFerguson incision, reconstruction of the surgical defect was performed using a free latissimusdorsi muscle flap. Twelve months after the

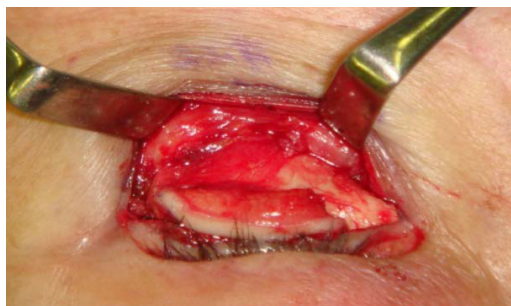

Figure 4. Cartilage was sutured and fixed to the bone membrane of the infraorbital rim.

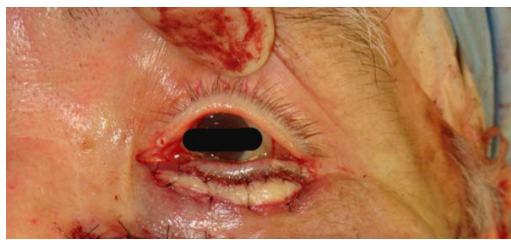

Figure 5. The composite graft was sutured

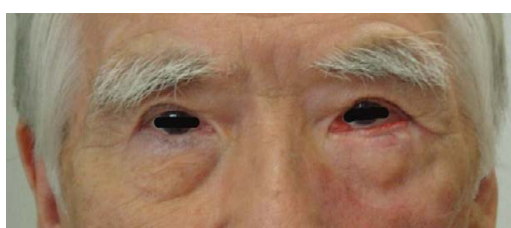

Figure 6. Nine months after the last correction.

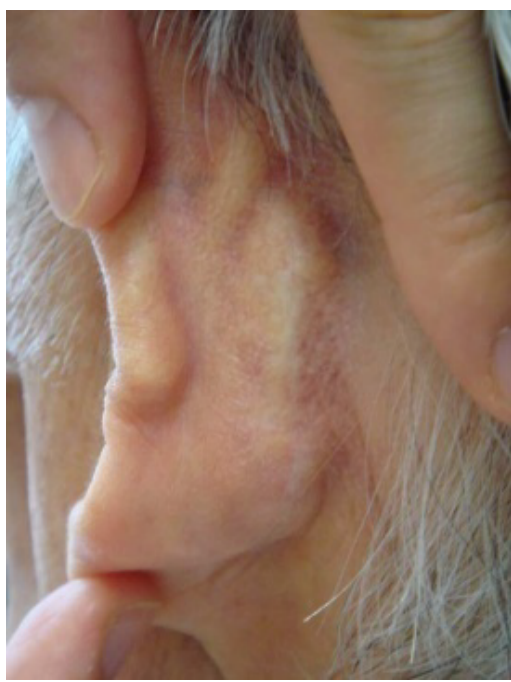

Figure 7. Scar at the donor site. 


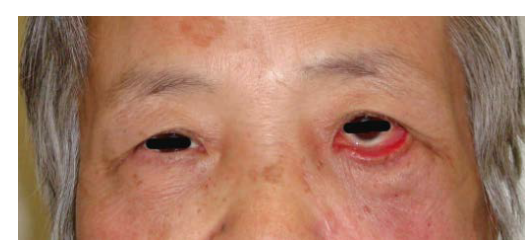

Figure 8. Preoperative case 2 .

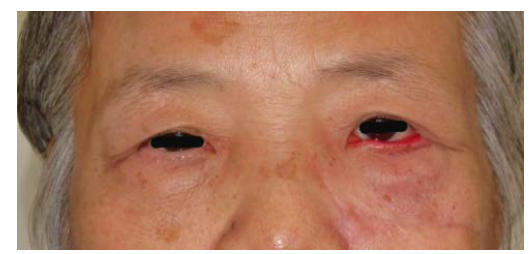

Figure 9. Six months after the first correction.

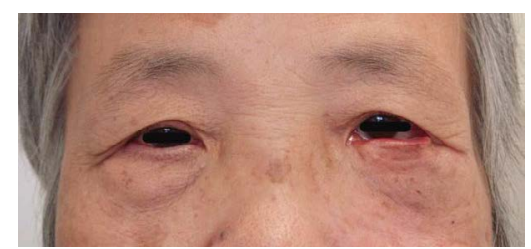

Figure 10. Twenty-one months after the second correction.

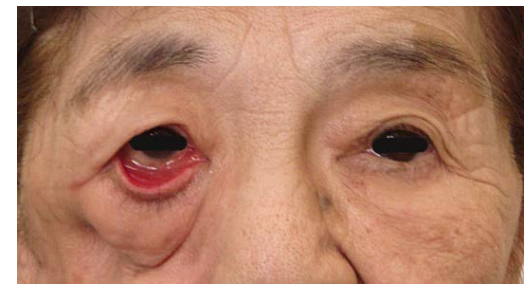

Figure 11. Preoperative case 2.

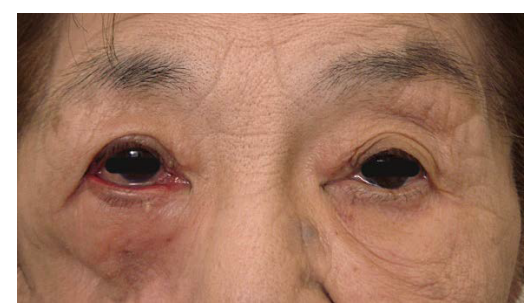

Figure 12. Five months after the correction.

operation, she developed ectropion and lagophthalmos (Figure 11). Therefore, auricular chondrocutaneous grafting was performed. A $20 \times 30 \mathrm{~mm}$ auricular cartilage graft combined with $5 \times 30 \mathrm{~mm}$ of cutaneous tissue was harvested and grafted to the lower eyelid in a manner similar to that described for Case 1.Ectropion improved after the operation (Figure 12).

\section{Discussion}

Deformities of the head and neck region can have serious effects on appearance and function of the patient and are among the most disabling and socially isolating defects. At Memorial Sloan-Kettering Cancer Center, resections of the maxilla for tumors have been classified into four basic types (1). Type 2 defects (subtotal maxillectomy) include resection of the maxillary arch, palate, anterior and lateral walls with preservation of the orbital floor (Figure 1)[1]. In this report, we made operation for 3 cases of ectropion of lower eyelids after subtotal maxillectomy (Type2). Ectropion is well known as one of the severe complications that can occur after surgical resection of maxillary cancers. Ectropion may be classified into the following five types (in decreasing frequency): involutional (senile), paralytic (neurogenic), cicatricial, mechanical, and congenital [6]. If the ectropion is mild, a lubricating ointment should be used for several days or weeks prior to ectropion repair.

The surgical strategy for correction of general ectropion depends on the specific etiology $[7,8]$. Horizontal lid laxity is usually corrected with a lateral tarsal strip procedure. Older methods include wedge resections and the Kuhnt-Szymanowski procedure. Whenever feasible, a lateral canthal tightening procedure is preferred. Tarsal ectropion requires reinsertion of the lower lid retractors, and augmentation of the anterior lamellae is required for cicatricialectropion. More severe cases of ectropion may need a skin flap or graft to lengthen the anterior lamella $[9,10]$.

In contrast, we think that ectropion after subtotal maxillectomy is mainly caused by 1) traction downward of soft tissue by contracture and weight of grafted flaps, 2) contracture by Weber-Ferguson incision and 3) dysfunction of orbicularis oculi muscle. Moreover, we found that the tarsus was soft and easy to bend during the operations in the present cases. This suggests that the cause of ectropion after maxillectomy might have been low vascularity of local tissue due to injury of the infraorbital artery after maxillectomy. Thus, the mechanism of onset of ectropion in these cases was likely different from those of simple facial nerve paresis, senile and congenital cases; this explains why the tarsal strip procedure and wedge resection were not effective in the present cases. Tightening of the lower lid in the context of a fragile tarsus can lead the eyelid downward along the curvature of eyeball.

When the cheek skin becomes weak, the skin can drop and result in stretching of the lower eyelid. For this deformity, a lamellar repair can be performed using composite grafts for the posterior lamella and skin for the anterior lamella. This strategy is effective in alleviating symptoms, and the reconstructed eyelids are stiff and immobile. Grafted skin becomes ultimately becomes inconspicuous, and the donor site was easy to suture. Only minor postoperative auricular deformity and scar were observed in this treatment.

The free graft of auricular cartilage is easy to prepare and adhere in high rate [2]. It is suitable for supporting the lower lid because it fits along the curvature of the eye ball by virtue of its thin, soft and pliable nature. The grafted cartilage lives on regional blood flow, so that it is possible to apply this method after waiting for recovery of the tissue damage after the radiation exposure. Additionally, it is reported that there is little deformation of grafted cartilage after radiation therapy [5]. These consequences establish that this method is appropriate for maxillary cancer cases.

This is the first study to describe lower lid ectropion after surgical resection of maxillary cancer. This type of complicated ectropion is difficult to correct via routine surgical means. We believe that a combined auricular cartilage/skin graft is material of choice for correction of ectropion that occurs after surgical resection of maxillary cancer operation. However, substructure is necessary to support the grafted cartilage, and enough tissue with blood flow is important for grafts surviving. In conclusion, our therapeutic strategy with subtotal maxillectomy cases is simple and appropriate for most cases, although it necessitates innovative surgery for cases with defects of the orbital rim and/or the eye lid tissue. 


\section{Conflict of Interest}

The authors declare that they have no conflict of interest.

\section{References}

1. Cordeiro PG, Santamaria E (2000) A classification system and algorithm for reconstruction of maxillectomy and midfacial defects. Plast Reconstr Surg 105: 23312346. [crossref]

2. Parodi PC, Calligaris F, De Biasio F, De Giovanna M, Flavia M, et al. (2013) Lower lid reconstruction utilizing auricular conchalchondral-perichondral tissue in patients with neoplastic lesions. A Biomed Res Int Epub 20: 1-6.

3. Rampazzo A, Gharb BB, Chi Chen H (2010) Total lower eyelid reconstruction with free posterior auricular chondrocutaneous flap. J Plast ReconstrAesthet Surg 163: 384-386.

4. Jackson IT, Dubin B, Harris J (1989) Use of contoured and stabilized conchal cartilage grafts for lower eyelid support:A preliminary report. Plast Reconstr Surg 3: 636-640. [corssref]
5. Hashikawa K, Terashi H, Tahara S (2007) Therapeutic strategy for the triad of acquired anophthalmic orbit. B Plast Reconstr Surg 119: 2182-2188. [crossref]

6. Neuhaus RW (1985) Anatomical basis of "senile" ectropion. Ophthal Plast Reconstr Surg1:87-89. [crossref]

7. Bedran EG, Pereira MV, Bernardes TF (2010) Ectropion. Semin Ophthalmol 25: 59-65 [crossref].

8. Miletic D, Elabjer BK, Bosner D, Busic M (2010) Our approach to operative treatment of lower lid ectropion. Acta Clin Croat 49: 283-287. [crossref]

9. Xue CY, Dai HY, Li L, Wang YC, Yang C, et al. (2012) Reconstruction of lower eyelid retraction or ectropion using a paranasal flap. Aesthetic Plast Surg 36: 611-617. [crossref]

10. Osborne SF, Eidsness RB, Carroll SC, Rosser PM (2010) The use of fibrin tissue glue in the repair of cicatricialectropion of the lower eyelid. Ophthal Plast Reconstr Surg 26: 409-412.[crossref]

Copyright: (C2016 Ochiai H. This is an open-access article distributed under the terms of the Creative Commons Attribution License, which permits unrestricted use, distribution, and reproduction in any medium, provided the original author and source are credited. 